\title{
压电材料的裂尖对数奇异性 $*$
}

\author{
秦庆华 余寿文 \\ (清华大学工程力学系, 北京 100084)
}

\section{关键词 压电材料 裂尖场 对数奇异性}

对于各向异性体平面问题, 其裂尖奇异性的研究常采用 Lekniskii 公式和 Stroh 公式, 如 弹性材料 ${ }^{[1,2]}$ 和压电材料 ${ }^{[3,4]}$. 与已有文献所得结果不同的是, 本文报道了压电介质中的裂尖对 数奇异性. 对于一均匀各向异性压电介质中的半无限长裂纹, 研究表明, 当 $r$ 趋于 0 时 ( $r$ 为裂 尖至考察点的距离), 其裂尖奇异性为 $r^{-1 / 2}$, 或为 $r^{-1 / 2} \ln r, r^{-1 / 2} \ln ^{2} r$ 和 $r^{-1 / 2} \ln ^{3} r$, 这取决于边界齐 次方程根的重数及重根性质.

\section{1 二维压电介质中的 Stroh 公式}

考虑一、二维压电介质, 假定所有场变量均只依赖于平面坐标, 如 $x_{1}$ 和 $x_{2}$. 问题的基本方 程为 ${ }^{[4]}$

$$
\begin{gathered}
\Pi_{i j, j}=0, \\
\Pi_{i j}=E_{i j k m} U_{k, m},
\end{gathered}
$$

式中

$$
\begin{gathered}
U_{k}=\left\{\begin{array}{cc}
U_{k}, & k=1,2,3, \\
\varphi & k=4 .
\end{array},\right. \\
\Pi_{i j}= \begin{cases}\sigma_{i j} & I=1,2,3, \\
D_{j} & I=4 .\end{cases} \\
E_{i j k m}= \begin{cases}c_{i j k m} & I, k=1,2,3, \\
e_{m i j} & I=1,2,3, k=4, \\
e_{j k m} & I=4, k=1,2,3, \\
-\varepsilon_{j m} & I=4, k=4,\end{cases}
\end{gathered}
$$

而 $\sigma_{i j}, U_{i}, D_{i}$ 和 $\varphi$ 分别为应力、弹性位移、电位移和电位势, $c_{i j k m}$ 为弹性刚度系数, $e_{i j k}$ 为压电系 系, $\varepsilon_{i j}$ 为介电常数.

对于压电方程 (1), 其一般解可表成 ${ }^{[4]}$

$$
\begin{aligned}
& U_{k}=A_{k} f(z), \\
& z=x_{1}+p x_{2},
\end{aligned}
$$

式中 $f$ 是一满足 (1) 式的解析函数, 而 $A_{k}$ 和 $p$ 由下面的特征方程确定 


$$
\begin{aligned}
& D_{I k} A_{k}=0, \\
& \left\|D_{I k}\right\|=0,
\end{aligned}
$$

式中 $\|\cdot\|$ 为求行列式, 而

$$
D_{l k}=E_{I|k|}+p\left(E_{I l k 2}+E_{l 2 k 1}\right)+p^{2} E_{l 2 k 2} .
$$

考虑到能量函数的正定性, 方程 (6) 不可能有实根, 我们将其 4 对共轭复根及其对应的特 征矢量取成

$$
\begin{gathered}
p_{k+4}=\bar{p}_{k}, \quad\left(\operatorname{Im}\left(p_{k}\right)>0\right), \\
A_{k+4}=\bar{A}_{k},
\end{gathered}
$$

式中 $\operatorname{Im}$ 表示取虚部, ( $)$ 表示复共轭. 若 $p_{k}$ 均为单根, 则问题的通解有如下形式:

$$
\begin{gathered}
U_{k}=\sum\left[A_{k J} q_{J} f\left(z_{J}\right)+\bar{A}_{k J} \bar{q}_{J} \bar{f}\left(z_{J}\right)\right], \\
\Pi_{l k}=\sum\left[B_{l k J} q_{J} f^{\prime}\left(z_{J}\right)+\bar{B}_{l k J} \bar{q}_{J} \bar{f}_{J}^{\prime}\left(z_{J}\right)\right],
\end{gathered}
$$

式中 ()$^{\prime}$ 表示对变量 $z_{J}$ 求导, $q_{J}$ 为一复常数. 这里和以后, 符号 “ $\sum$ ”均表示对指标 $J$ 由 1 到 4 求和, 而 $B_{l k L}$ 为

$$
B_{l k L}=\left(E_{l k J l}+p_{L} E_{l k J 2}\right) A_{J L} \quad(L \text { 不求和 }) .
$$

\section{2 裂尖场的对数奇异性}

\section{1 裂尖场的一般解}

考虑一沿 $x_{1}$ 轴负向的半无限长裂纹, 其试解可取成

$$
f(z)=\frac{1}{1-\eta} z^{1-\eta}
$$

将 (13) 式代人 (10) 和 (11) 式, 有

$$
\begin{gathered}
U_{k}=\sum\left[A_{k J} q_{J} z_{J}^{1-\eta}+\bar{A}_{k J} \bar{q}_{J} \bar{z}_{J}^{1-\eta}\right] /(1-\eta), \\
\Pi_{l k}=\sum\left[B_{l k J} q_{J} z_{J}^{-\eta}+\bar{B}_{J k} \bar{q}_{J} \bar{z}_{J}^{-\eta}\right] .
\end{gathered}
$$

由 (15) 式可看出, 应力和电位移 $\Pi_{I J}$ 的奇异阶数为 $r^{-\eta}$. 若 $\eta$ 的实部大于零, 则应力和电位移 是奇异的. 另一方面, 为使裂尖位移和电位势为有限值, 又要求 $\operatorname{Re}(\eta)<1$. 故应取 $0<\operatorname{Re}(\eta)<1$. 设在裂纹面不受力和自由电荷作用, 则边界条件可写成

$$
\begin{gathered}
\Pi_{I 2}(\pi)=-r^{-\eta}\left(\mathrm{e}^{-\mathrm{i} \pi \eta} B q^{\mathrm{T}}+\mathrm{e}^{\mathrm{i} \pi \eta} \bar{B} \bar{q}^{\mathrm{T}}\right)=0, \\
\Pi_{I 2}(-\pi)=-r^{-\eta}\left(\mathrm{e}^{\mathrm{i} \pi \eta} B q^{\mathrm{T}}+\mathrm{e}^{-\mathrm{i} \pi \eta} \bar{B} \bar{q}^{\mathrm{T}}\right)=0,
\end{gathered}
$$

或矩阵形式

$$
K Q=0,
$$

式中 $(B)_{I J}=B_{I J}, q=\left\{q_{1} q_{2} q_{3} q_{4}\right\}, Q=\{q \bar{q}\}^{\mathrm{T}}$, 对于 $Q$ 的非平凡解, 必使

$$
\|K\|=0 \text {. }
$$

由矩阵 $B$ 的非奇异性, (19) 式给出

$$
\left(1-\mathrm{e}^{4 i \pi \eta}\right)^{4}=0 .
$$

由此解得

$$
\eta=(1-n) / 2 \quad(n=0,1,2, \cdots) .
$$

因需满足 $0<\operatorname{Re}(\eta)<1$, 应取 $n=0$, 它是 (20) 式的一个 4 重根. 将 $\eta=1 / 2$ 代人 (14) 和 (15) 式 得 


$$
\begin{aligned}
& U_{I}=4 \operatorname{Re} \sum r^{1 / 2} A_{I J} q_{J}\left(\cos \theta+p_{J} \sin \theta\right)^{1 / 2}, \\
& \Pi_{I K}=2 \operatorname{Re} \sum r^{-1 / 2} B_{I k J} q_{J}\left(\cos \theta+p_{J} \sin \theta\right)^{-1 / 2} .
\end{aligned}
$$

\section{$2.2 p$ 为重根时的修正解}

以上讨论是在 $p$ 为单根前提下进行的. 若 $p$ 为重根, 则通解 (3) 中的 8 个函数将不完全独 立, 需另外寻找补充解 ${ }^{[S]}$. 首先我们考虑 $p_{i}$ 为双重根情形. 这时 (10) 和 (11) 式仍为问题的一 组解,而所需的补充解为

$$
\begin{gathered}
U_{l}^{(2)}=4 \operatorname{Re} \sum r^{1 / 2} q_{J} \frac{\mathrm{d}}{\mathrm{d} p_{i}}\left[A_{I J}\left(\cos \theta+p_{J} \sin \theta\right)^{-1 / 2}\right], \\
\Pi_{l k}^{(2)}=2 \operatorname{Re} \sum r^{-1 / 2} q_{J} \frac{\mathrm{d}}{\mathrm{d} p_{i}}\left[B_{l k J}\left(\cos \theta+p_{J} \sin \theta\right)^{-1 / 2}\right],
\end{gathered}
$$

式中 $\mathrm{d} A_{l J} / \mathrm{d} p_{i}$ 可按下式求得:

$$
\left.\frac{\mathrm{d}}{\mathrm{d} p_{i}}\left(D_{l k} A_{k}\right)\right|_{p=p_{i}}=0
$$

而 $\mathrm{d} B_{l k J} / \mathrm{d} p_{i}$ 可通过微分 (12) 式而得.

线性齐次方程组 (5) 和 (26) 有非零解的条件为 ${ }^{[6]}$

$$
\frac{\mathrm{d}^{n}}{\mathrm{~d} p^{n}}\left\|D_{l k}\right\|_{p=p_{i}}=0,(n=N-M),
$$

式中 $N$ 和 $M$ 分别为矩阵 $D_{I k}$ 的阶和秩. 然而, 我们发现, 解的奇异阶并未因对 $p$ 求导而改 变.

类似地, 可得到 $p$ 为三重根或四重根时的补充解.

\section{$2.3 \eta$ 为重根时的修正解}

如果 $\eta$ 为 (19) 式的重根, 则 $q_{J}$ 不完全独立, 故也需另找独立解. 当重数为 $m$ 时, 所需的 $m-1$ 个补充解为 (以 $\Pi_{l k}$ 为例)

$$
\Pi_{l k}^{(i)}=2 \operatorname{Re} \sum B_{l k J} z_{J}^{-\eta}\left(-\ln z_{J}+\frac{\partial}{\partial \eta}\right)^{i} q_{J} \quad(i=1,2, \cdots, m-1),
$$

同样地, 这些解的存在性取决于下式:

$$
\frac{\mathrm{d}^{n}}{\mathrm{~d} \eta^{n}}\|K\|=0, \quad n=8-M
$$

是否成立, 式中 $M$ 为 $K$ 的秩. 在本文中, 既然 $\eta$ 为边界方程 (18) 的 4 重根, 应力和电位移在 裂尖的奇异性就必为下述 4 种情形之一:

$$
\Pi_{I J}(r)=\left\{\begin{array}{lll}
\mathrm{O}\left(r^{-1 / 2}\right) & \text { 只满足 } & K Q=0, \\
\mathrm{O}\left(r^{-1 / 2} \ln r\right) & \text { 满足 } & \left.\frac{\mathrm{d}}{\mathrm{d} \eta}(K)\right|_{\eta=1 / 2}=0, \\
\mathrm{O}\left(r^{-1 / 2} \ln ^{2} r\right) & \text { 满足 } & \left.\frac{\mathrm{d}^{2}}{\mathrm{~d} \eta^{2}}(K)\right|_{\eta=1 / 2}=0, \\
\mathrm{O}\left(r^{-1 / 2} \ln ^{3} r\right) & \text { 满足 } & \left.\frac{\mathrm{d}^{3}}{\mathrm{~d} \eta^{3}}(K)\right|_{\eta=1 / 2}=0 .
\end{array}\right.
$$




\section{参考文献}

1 Sou Z. Singularities, interfaces and cracks in dissimilar anisotropic media. Proc R Soc Lond, 1990, A427: $331 \sim 358$

2 Ting T C T. Explicit solution and invariance of the singularities at an interface crack in anisotropic composites. Int J Solids Structures, 1986, 22: 956 983

3 Liang J, Han J, Du S. Rigid line inclusions and cracks in anisotropic piezoelectric solids. Mech Res Commu, 1995, $22(1): 43 \sim 49$

4 Pak Y E. Linear electro-elastic fracture mechanics of piezoelectric materials. Int J Frac, 1992, 54: 79 100

5 Ting T C T. Effects of change of reference coordinates on the stress analysis of anisotropic elastic materials. Int $\mathbf{J}$ Solids Structures, 1982, 18: 139 152

6 Dempsey J P, Sinclair G B. On the stress singularities in the plane elasticity of the composite wedge. J of Elasticity, 1979, $9(4): 373 \sim 391$ 